\title{
A Study on Cross Layer MAC design for performance optimization of routing protocols in MANETs
}

\author{
P.K.Alima Beebi \\ School of Information Technology and \\ Engineering, \\ VIT University, Vellore. 632014, \\ Tamil Nadu, India. \\ Email:haleemamca@gmail.com
}

\author{
Sulava Singha \\ School of Information Technology and \\ Engineering, \\ VIT University, Vellore. 632014, \\ Tamil Nadu, India. \\ Email: sulava_it@gmail.com
}

\author{
Ranjit Mane \\ School of Information Technology and \\ Engineering, \\ VIT University, Vellore. 632014, \\ Tamil Nadu, India. \\ Email:maneranjit@gmail.com
}

\begin{abstract}
One of the most visible trends in today's commercial communication market is the adoption of wireless technology. Wireless networks are expected to carry traffic that will be a mix of real time traffic such as voice, multimedia conferences, games and data traffic such as web browsing, messaging and file transfer. All of these applications require widely varying and very diverse Quality of Service (QoS) guarantees. In an effort to improve the performance of wireless networks, there has been increased interest in protocols that rely on interactions between different layers. Cross-Layer Design has become the new issue in wireless communication systems as it seeks to enhance the capacity of wireless networks significantly through the joint optimization of multiple layers in the network. Wireless multihop ad-hoc networks have generated a lot of interest in the recent past due to their many potential applications. Multi-hopping implies the existence of many geographically distributed devices that share the wireless medium which creates the need for efficient MAC and routing protocols to mitigate interference and take full advantage of spatial reuse. Cross-Layer Design is an emerging proposal to support flexible layer approaches in Mobile Ad-hoc Networks (MANETs). In this paper, we present few Cross-Layer MAC design proposals by analyzing the ongoing research activities in this area for optimizing the performance of routing protocols in MANETs.
\end{abstract}

Keywords- cross-layer, routing, MAC, MANET, multi-hop networks;

\section{INTRODUCTION}

An ad-hoc network is a local area network (LAN) that is built spontaneously as devices connect. It is self-creating, selforganizing and self-administrating network. Each node acts as a host and router and forwards each other's packet to enable the communication between nodes. The network topology changes frequently because of node mobility and power limitations. Multi-hopping in ad-hoc networks implies the existence of many geographically distributed devices that share the wireless medium. Efficient routing is the fundamental issue in multi-hop wireless ad-hoc networks $[1,2]$.

A layered architecture, like the seven-layer open systems interconnect (OSI) model [3, p.20], divides the overall networking task into layers and defines a hierarchy of services to be provided by the individual layers. The services at the layers are realized by designing protocols for the different layers. The architecture forbids direct communication between non adjacent layers; communication between adjacent layers is limited to procedure calls and responses. It is repeatedly argued that although layered architectures have served well for wired networks, they are not suitable for wireless networks. The complexity and time-varying attributes of the wireless channel call for cross-layer design [4]. Protocols can be designed by violating the reference architecture, for example, by allowing direct communication between protocols at nonadjacent layers or sharing variables between layers. Such violation of a layered architecture is called cross-layer design with respect to reference architecture [5].

It is argued that while designing efficient routing protocols for multi-hop wireless ad-hoc networks to meet the QoS requirements, we also need to consider the influence of MAC protocol in finding the optimal routes. Researchers analyzed the interaction between routing and the MAC layer protocols and confirmed that the MAC protocols can significantly affect the performance of routing protocols and vice versa.

Thus, a central challenge in the design of ad-hoc networks is the development of efficient MAC and dynamic routing protocols that can efficiently find routes between the communicating nodes. In this paper, we focus on cross layer design proposals based on the coupling between network layer and MAC layer for optimizing the performance of routing protocols in MANETs.

Widely used routing protocols for Ad-hoc networks are the two major categories:

\section{A. Pro-active (table-driven) Routing Protocols:}

This type of protocols maintains fresh lists of destinations and their routes by periodically distributing routing tables throughout the network.

The DSDV and OLSR are well-known proactive routing protocols.

\section{B. Reactive (on-demand) Routing Protocols:}

This type of protocols finds a route on demand by flooding the network with Route Request (RREQ) packets. 
The AODV and DSR are representatives of on-demand routing protocols.

The fundamental MAC technique of IEEE 802.11 based WLAN standard is the Distributed Coordination Function (DCF). DCF employs a CSMA/CA with Binary exponential back-off algorithm. This algorithm is used to space out repeated retransmissions of the same block of data, often as part of network congestion avoidance.

\section{AN OVERVIEW OF THE PROPOSALS}

The layered architecture can be violated by creating new interfaces between the layers or by merging of adjacent layers [5]. The new interfaces are used for information sharing between the layers at runtime. Depending on the direction of information flow along the new interfaces, we classify the different proposals in the literature under consideration into the following categories:

1. The cross-layer proposals in which some information from the MAC layer is passed onto network layer for optimized routing in MANETs.

2. The cross-layer proposals in which some parameter of the network layer is passed onto the MAC layer to improve the performance of routing protocols.

3. The cross-layer proposals in which some information is passed back and forth between the two layers for efficient routing.

The adjacent layers are merged by designing a new sublayer such that the services provided by the new sublayer is the union of the services provided by the constituent layers.

4. Novel routing protocols based on cross-layer coupling between MAC and network layer.

The route maintenance overhead in AODV increases as the network mobility is high and the topology changes frequently. The established routes are to be changed as the nodes move away. It results in low performance as many packets are dropped when some active router node on the path moves away significantly. The performance of AODV protocol is improved by adopting a cross-layer approach and position based forwarding technique [6]. Here the MAC layer calculates the received power of the packets from the nodes and informs network layer if it is below the threshold value for efficient transmission. (Category 1). The network layer removes those nodes from the routing table and finds an alternate path.

\section{A. Implementation of AODV-PF:}

AODV-PF is an update over on-demand routing protocol.
In position-based forwarding, every node maintains a list of neighbors nearest to the position of the destination. At the time of route establishment, only these nodes will be selected. Hence the route lifetime improves. A forwarding region is either a circular or a Box like virtual area drawn around the destination node.

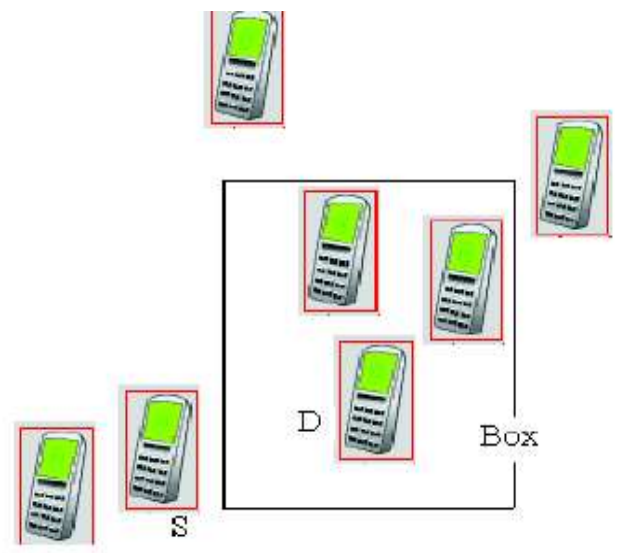

Figure 1: Sample PF Box forwarding region. A route between source node $S$ and destination node $D$ is found by flooding the forwarding region.

When a node, $S$, needs a route to a destination, $\mathrm{D}$, it floods a route request (RREQ) through the forwarding region/ entire network attempting to find the destination. In PF Box, a neighbor of $\mathrm{S}$ determines whether it is within the forwarding zone by using the location of $\mathrm{S}$ and the expected zone for $\mathrm{D}$.

Once the path is established, source transmits data packets to the destination. There may be some nodes which may be in the same direction as of the destination, but may not have sufficient energy to forward the packets further. Such nodes must notify their predecessors about the energy constraint. Energy is a physical layer feature, which is measured at the MAC layer. If the estimated energy is below the threshold value for efficient transmission, then the routing layer is notified about the energy fall. Accordingly routing layer initializes route maintenance by notifying its neighbors about the problem and removes those nodes from the routing table.

AODV-PF outperforms on-demand routing protocols (AODV) in various constraints such as control overhead, throughput, latency when simulated with pause time and different loads. AODV-PF sustains high packet delivery rates. In terms of routing overhead, AODV-PF has scalable routing overhead for mobility, random packet loss, and traffic load, thus utilizing the channel efficiently.

A strategy of cross-layer algorithm AODV- SPF (Scheduling Priority Flow) is proposed in [7] to address the Intra-Flow contention problem in chain topology where the source, could actually inject more packets into the chain than the subsequent nodes can forward. 


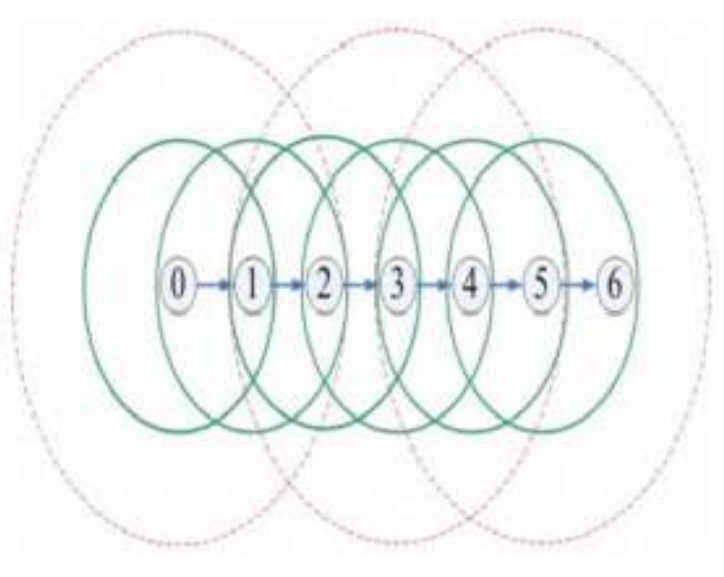

Figure 2: MAC layer interference among a chain topology. Small circle denotes node's valid transmission range. Large circles denote node's interference range. Node 0 is the source, 6 is the destination.

These packets are eventually dropped at the two subsequent nodes. In the shared channel environment of multi-hop ad hoc network, intra-flow contention is widespread and result in collision and congestion at some nodes. This not only greatly decreases the end-to-end throughput but also increase the probability of link failure during data transmission in network layer. Here the total hop count parameter is transmitted from network layer to MAC layer (Category 2) which is used to recalculate the contention window of the nodes along the routing path. This approach avoids collision in the MAC layer and results in getting better performances of data transmission in the network layer.

\section{B. Implementation of $A O D V-S P F$ :}

The AODV-SPF includes two major mechanisms. The first one is to assign a higher probability of channel access to the downstream node. This could achieve optimum packet scheduling for chain topology and avoid severe intra-flow contentions in each flow. The second one is to limit the data/fragment outgoing rate of source node in MANET, not to allow the source node to occupy the whole outgoing queue and bandwidth. This could efficiently prevent the irresponsible applications from injecting more packets than the network could handle, and leave more queue space and bandwidth for other flows passing through network, which further alleviates the unfairness problem between moderate and greedy source flows. One way to prevent the first node on the path from injecting more packets than what the succeeding nodes can forward is to assign the lowest channel access probability to source node and higher channel access probability to intermediate nodes along the downstream path. This can achieve optimum scheduling for one-way traffic in the regular chain topology.

Extensive simulations verify that compared to IEEE 802.11 DCF, this scheme, AODV -SPF, in most of the cases could achieve better performance metrics of data transmission in network layer, e.g., stable and higher throughput, packet delivery ratio, lower normalized routing load, decreasing the number of control messages such as Route Request and Route Error.
A mobile station that experiences bad channel tends to transmit at a low rate in order to decrease the bit error rate (BER). In [8], a cooperative MAC protocol is used to improve the performance of the routing protocol (DSDV) in the network layer. In the cooperative MAC protocol, a station would use a neighboring helper station for MAC layer forwarding, if the two-hop relaying yields to a better performance than a direct single-hop transmission. A cross-layer approach is followed here where the DSDV routing protocol finds a multi-hop path from the source to destination while the cooperative MAC scheme, eventually selects two-hop forwarding for each routing layer hop (category 3 ), to boost the performance of the routing protocol.

\section{Implementation of Cooperative Routing MAC protocol:}

Every station maintains information about its candidate helpers in a table called CoopTable. Corresponding to a particular helper station, each row in the CoopTable stores the MAC address of the helper and the transmission rates that this helper could provide for the two hop transmission (i.e., from the transmitter to the helper, and from the helper to the intended destination).

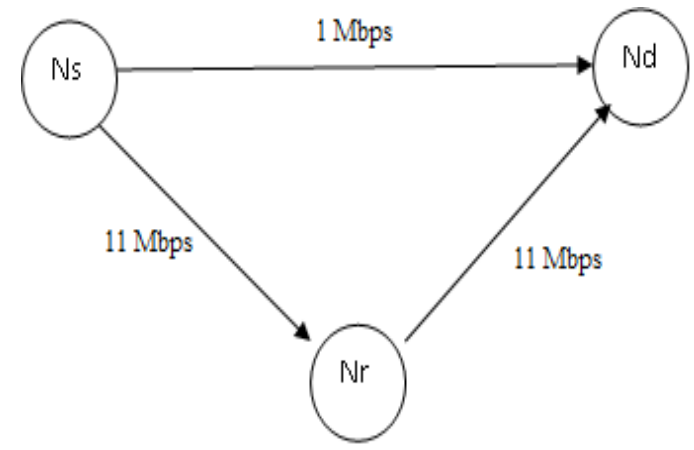

Figure 3: Represents a simple network consisting of a source node (Ns ), a destination node $(\mathrm{Nd})$, and a helper node $(\mathrm{Nr})$.

In a real environment, every station could be considered as a candidate helper by its neighboring stations. The authors have implemented a broadcasting scheme using a hello packet in each station. The hello packet is generated directly by the MAC layer and is broadcasted on a periodic basis, and it indicates the sustainable rates between the particular station and its neighbors. A mobile station updates its CoopTable based upon the received Hello messages, in order to be aware of candidate helpers, and revokes timely an enlisted helper once the helper becomes inactive.

The extensibility of the cooperative MAC protocol into multi-hop ad-hoc networks, where in conjunction with the routing protocol can achieve superior performance, compared to the legacy $802.11 \mathrm{~g}$.

Due to the highly complicated nature of medium access control (MAC) layer in wireless networks, MAC protocol has been implemented as software. This is different from a wired network situation where MAC is implemented in hardware. Due to the software implementation of MAC protocol, the traditional routing structure in multi-hop wireless ad hoc networks results in long processing delays for forwarding packets in every intermediate/relay node. The authors in [9] 
propose a solution to alleviate this issue based on cross-layer MAC design, which improves the coordination between MAC and routing layers using an idea called "virtual link". Experimental results show that the proposed cross-layer design significantly improves the performance in terms of reduced round trip time(RTT), reduced processing time in the intermediate relay/forwarding nodes and increased throughput compared to a legacy architecture.

\section{Implementation of cross layer MAC enabling Virtual Link:}

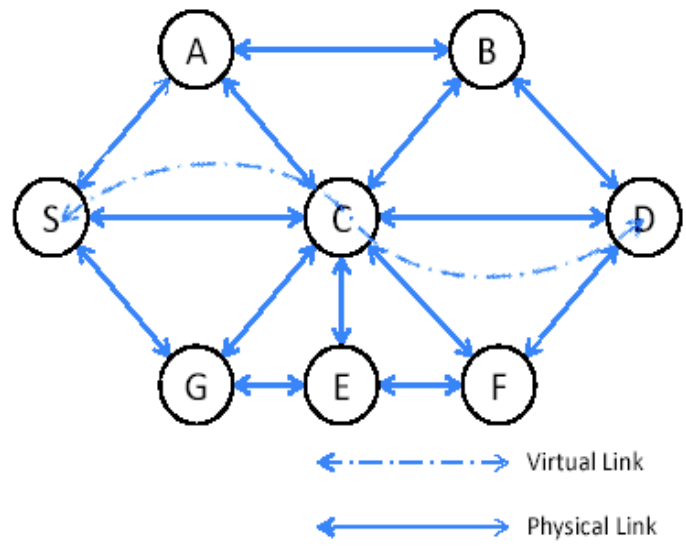

Figure 4: A multi-hop ad-hoc network example

In the proposed cross-layer MAC architecture, the authors introduce two extra modules: Inbound Monitor module and Self-Learning module. The steps for creating a virtual link are then as follows. When the wireless MAC starts to run in a node, its IP address is noted and the Inbound Monitor also starts to run. The Inbound Monitor of this node checks for the destination IP address on each frame. If the destination IP is equal to its own IP address, this is treated as a normal frame. Otherwise, the Inbound Monitor will look up the corresponding virtual link entry for this frame. If a suitable virtual link is located successfully, this frame will be re-encapsulated according to this virtual link entry and sent to the physical layer immediately for relay/forwarding purpose.

If no corresponding virtual link is found, the self-learning module will be triggered. From now on, this monitor module will work on the outbound direction of the IEEE 802.11 MAC. After routing layer re-encapsulates the frame, which triggers the self-learning module, this frame will be shown again on the outbound direction. The self-learning module will create a suitable virtual link according to the new MAC header of this frame. When other data frames arrive at this node, the Inbound Monitor will re-encapsulate the MAC header according to corresponding Virtual Link entry.

The tests performed clearly demonstrate that cross-layer MAC design employing the proposed virtual link concept reduces the processing time at the intermediate nodes approximately by $50 \%$ while the throughput increases by $7-$ $10 \%$ when compared with the legacy routing algorithm.

LEss remaining hop More Opportunity (LEMO) algorithm was proposed to improve the packet delivery ratio and fairness among flows for multi-hop ad hoc networks through crosslayer interaction between MAC and the routing layer. The routing information about the total hops and the remaining hops required by a packet to reach its destination is exploited by the MAC layer (Category 2) in order to give priority to the packets that are closer to their destination. Reference [10] compares the performance of LEMO algorithm by using DSR and AODV protocols at the routing layer and varying the mobility and the load conditions. With the help of performance metrics like packet delivery ratio, average end-to-end delay and normalized routing load, it is shown that cross-layering between DSR and IEEE 802.11 DCF performs better than cross-layering between AODV and IEEE 802.11 DCF.

\section{E. Implementation of LAODV and LDSR:}

LAODV algorithm is implemented by applying crosslayered approach between AODV protocol at the routing layer and IEEE 802.11 DCF at the MAC layer. In order to achieve this, the information about the total number of hops between the source and the destination nodes, and the number of remaining hops from the forwarding node is collected from the routing layer and is sent to the MAC layer. The IEEE 802.11 DCF used at the MAC layer is modified to process the received information from the routing layer and change the value of $C W m i n$ accordingly. LDSR algorithm is implemented in a similar manner by using DSR as the routing layer protocol instead of AODV.

It is concluded from the simulation results that LAODV and LDSR have better packet delivery ratio. Both have shown significant improvement in performance in terms of average end-to-end delay and normalized routing load. Cross layering between DSR and IEEE 802.11 DCF has shown better results than cross-layering between AODV and IEEE 802.11 DCF. LDSR has shown an increase in packet delivery ratio up to $2 \%$ whereas with LAODV marginal increase can be seen.

In [11], the authors propose a novel Cross-layer Synchronous Dynamic Token Protocol (CLSDTP) in single channel that is based on token-passing scheme (Category 4). The protocol introduces a token relay algorithm which is fast and adaptive to topology variation, presents a collision avoidance algorithm which solves the exposed and hidden terminal problem. The CLSDTP improves the spatial multiplexing compared with the RTS/CTS access mechanism. The results of the simulation show that CLSDTP can significantly improve the system performance.

\section{F. Implementation of CLSDTP:}

In CLSDTP, the Time Slot is defined as the time need to send a token and a data packet. Every node synchronizes with each other through monitoring messages transferred by its neighbors. A node begins to transfer token at the time T0, send data packet at the time T1. Node sends one data packet in one time slot. If a node hears a token transferred by its neighbor, it will realize it is the beginning of a slot. If a node hears a data packet sent by its neighbor, it will realize it is the time $\mathrm{T} 1$ of a slot. There should be no worry about the timeslot synchronization because the Time Slot is long and the guard interval. Same as WDTP, each node should maintain a token passing queue (TPQ) to record its neighbor nodes. The nodes not holding the token listen to the channel. If they find a node processed the token transfer, they push the node to the rear of 
their TPQs. The node holding the token transfers the token to the front node in its TPQ. Successful token transmission relies on implicit acknowledgment which is the successor's token transmission. A node will consider that the connection with its successor is broken, if the successor does not transfer token in the beginning of the next timeslot, which indicates that the transfer was unsuccessful. It should delete the node from its TPQ and transfer the token again to the new front node of its TPQ.

The most important feature of CLSDTP is the sharing of net information by MAC and routing layer, which reduces the system overhead significantly. The protocol inherits the advantage of the token passing scheme under which the probability of collision is very low, which effects the performance of network dramatically. The results of Spatialmultiplexing analysis and the simulation demonstrate that CLSDTP outperforms 802.11 RTS/CTS-AODV protocol in terms of system throughput and delay.

A novel cross-layer efficient routing protocol (CLERP) is presented in [12]. CLERP adopts cross-layer design to establish backup route to reduce the packet losses when link breaks occur. To decrease the unnecessary overhead of hello packets, adaptive links connectivity is employed to improve connectivity and coverage when the nodes are far away from the primary route. The simulation results demonstrate that CLERP yields lower route discovery frequency, higher packet delivery fraction, better average end-to-end delay and lower routing load.

\section{G. Implementation of CLERP:}

CLERP is presented by sharing the cross-layer cache information while still maintaining separation between the MAC layer 802.11 and the route layer AODV in protocol design. Cross-layer cache is used to enhance the connectivity of the network. Node updates its cross-layer cache if any communication is heard from any neighbor (Category 1). If a node receives any messages from its neighbors, the neighbors' link status is set to active and the timeout is reset to the current time plus active timeout. If active timeout passes without any messages from a neighbor, the neighbor's link status changes to inactive. Once in the inactive state, if there is still no sign of the neighbor during the delete interval, the neighbor is deleted from the cross-layer cache. Cross-layer cache can be used to establish backup route to reduce the packet losses due to link break. The backup routes are established during the RTS/CTS/DATA/ACK exchange procedure. When a node that is not part of the route overhears RTS messages transmitted by a neighbor, it records MAC address of the receiver and the sender of the packet. When the node overhears the CTS messages, it checks if the recorded sender of the RTS is the receiver of the CTS. If it is, the receiver of the RTS is a neighbor and should be inserted in the cross-layer cache. Meanwhile, it records that neighbor as the next hop to the destination in its backup route table. Using this method, backup route can be conducted. When a node detects a link break, it caches the packets transmitted to it and then broadcasts a one hop Route Request (RREQ) to candidate for backup routes. After receiving this RREQ, nodes that have a routing to the destination in their alternate route table, forward the packet to their next hop node. Data packets therefore can be delivered through one or more alternate routes and are not dropped when link breaks occur. If no backup route can be constructed with the downstream node, an RERR message is propagated toward the source node to initiate a route rediscovery after a timeout value.

Simulation results prove that CLERP increases the packet delivery fraction, reduces the route discovery frequency, average end-to-end delay and normalized routing load.

\section{CONCLUSION AND FUTURE RESEARCH}

In accordance with the review performed, we propose to combine the strategies followed in more than one literature in the following manner to further improve the performance of the routing protocols in MANETs. 1. AODV-PF and AODV-SPF can be combined with the Cooperative Routing MAC protocol to further improve the performance of routing protocols. 2 . AODV-SPF considers only the chain topology. We can focus on how this AODV-SPF can be extended on networks with other topologies and can analyze the results through simulation. 3 . To reduce packet processing delay at every node the concept of virtual link can be incorporated along with other strategies for performance improvement of routing protocols. 4. AODVSPF can be combined with LEMO algorithm to further improve the performance of routing protocols in MANETs. We also suggest modifying the existing approach in the literatures with the new one and analyzing whether it could be possible to bring out the best outcome. 5. In CLSDTP, a token passing scheme is used through which MAC and network layer share net information. This idea of token passing can be combined with AODV-PF to pass information about the weak nodes to network layer. In CLERP, AODV is used at the routing protocol in the network layer. Instead other on-demand routing protocol such as DSR can be used and the results can be analyzed through simulation. Also we plan to introduce more parameters other than the existing parameters and try to analyze the results through simulation.

\section{REFERENCES}

[1] Carine TOHAM, François JAN "Multi-interfaces and Multi-channels Multi-hop Ad hoc Networks: Overview and Challenges" (MASS), 2006 IEEE International Conference on Oct. 2006, Print ISBN-1-4244-05076.

[2] Catherine Rosenberg "Challenges in Multi-Hop Networks" Next generation internet design and Engineering, 2006. NGI, $20062^{\text {nd }}$ Conference on. E-ISBN-0-7803-9456-9. Print ISBN-0-7803-9455-0.

[3] D.Bertsekas and R.Gallager, Data Networks, $2^{\text {nd }}$ ed., Prentice Hall, 1992.

[4] Frank Aune , "Cross-Layer Design Tutorial" Published under Creative Commons License.D.Bertsekas and R.Gallager, Data Networks, $2^{\text {nd }}$ ed., Prentice Hall, 1992.

[5] Vineet Srivastava, Mehul Motani "Cross-Layer Design: A survey and the road ahead" Communication Magazine, IEEE. Issue date: Dec, 2005. ISSN: 0163-6804.

[6] Patil R.; Damodar A.; Das R. "Cross Layer AODV with Position based Forwarding Routing for Mobile Adhoc Network MANET" Wireless Communication and Sensor Networks $20095^{\text {th }}$ IEEE Conference on 10.11.09/ACSN,2009.5434796. Print ISBN-978-1-4244-5876-9.

[7] So tsung Chou; Hann Tzong Chun; Chang-Ho Shiao; "AODV Protocol with Scheduling Priority Flow in Mac Layer (AODV - SPF)" Networked Computing and Advanced Information Management (NCM), $20106^{\text {th }}$ International Conference on Publication year 2010. Issue date: 16-18 Aug 2010. Print ISBN: 978-1-4244-7671-8.

[8] Jian Lin, Thanasis Korakis, Xiao Wang, Shunyuan Ye and Shivendra Panwar "A Demonstration of a Cross-Layer Cooperative Routing-MAC 
scheme in Multi-hop Ad-Hoc Networks" Testbeds and Research infrastructure for the Development of Network and Communities and workshops, 2009. TridentCom $20095^{\text {th }}$ International Conference on Issue date: 6-8 April 2009. ISBN: 978-1-4244-2846-5.

[9] Kai Hong Shamik Sengupta R. Chandramouli "Cross-layer MAC Enabling Virtual Link for Multi-hop Routing in Wireless Ad-Hoc Networks" Communications (ICC), 2010 IEEE International Conference on Issue date: 23-27 May 2010. ISSN: 1550-3607. Print ISBN: 978-14244-6402-9.

[10] Manjul Walia and Rama Krishna Challa "Performance Analysis of Cross-Layer MAC and Routing Protocols in MANETs" Computer and Network Technology (ICCNT), $20102^{\text {nd }}$ International Conference on Issue date: 23-25 April 2010. Print ISBN: 978-0-7695-4042-9.

[11] Yao Chang-hua, Wang Cheng-gui "A Cross-Layer Synchronous Dynamic Token Protocol for Ad-Hoc Networks" Communication and Mobile Computing (CMC), 2010 International Conference on 12-14 April 2010. Vol. 3 Print ISBN: 978-1-4244-6327-5.

Wang Qing-wen, Shi Hao-shan, Jiang Yi, Cheng Wei "A Cross-Layer Efficient Routing Protocol for Ad hoc Networks" Mobile Adhoc and Sensor Systems, 2009. Mass'09, IEEE $6^{\text {th }}$ International Conference on Issue date: 12-15 Oct 2009. Print ISBN: 978-1-4244-5113-5.

\section{AUTHORS PROFILE}

P.K.ALIMA BEEBI is a M.Tech(Networking) student in the IT department at Vellore Instiutute of Technology, Vellore, TamilNadu, India. Her research interest is in distributed database systems, wireless communications and networking. She holds an M.C.A. degree from Madurai Kamaraj University, TamilNadu, India and M.Phil degree from Periyar University, India. Her M.Phil dissertation is on "Emerging Database Technologies and its Applications".

SULAVA SINGHA is a M.Tech(Networking) student in the IT department at Vellore Instiutute of Technology, Vellore, TamilNadu, India. Her research interest is in distributed operating systems and networking. She holds an B.Tech degree in Information Technology from Pune University, India.

RANJIT MANE is a M.Tech(Networking) student in the IT department at Vellore Instiutute of Technology, Vellore, TamilNadu, India. 